\title{
RULES FOR THE CHEMISTRY LABORATORY OR CLASSROOM
}

\author{
Iwona Maciejowska, Jaroslaw Wilanowski \\ Faculty of Chemistry, Jagiellonian University, Krakow, Poland
}

\section{A. General}

1. You are allowed in science laboratories only in the presence of a teacher. You must never enter a preparation room without an explicit invitation.

2. During practical classes you must follow your teacher's instructions to the letter.

3. You may carry out experiments only with the explicit permission of your teacher.

4. Never taste anything in the laboratory, or put anything in your mouth. Only touch or smell chemicals with the approval of your teacher.

5. It is your responsibility to keep your personal workplace tidy. Only items needed for the work in hand should be there. Bags and coats must not become trip hazards.

6. Eating or drinking is not allowed in the chemistry classroom. Food or drink must not be placed on the benches or tables.

7. Never take chemicals, other materials and equipment out of the laboratory without the permission of your teacher.

8. If you notice any broken or defective equipment or glassware, report this to your teacher immediately.

9. In the event of an accident or fire, alert your teacher. (You should know where the safety equipment is located in the laboratory/classroom: first-aid kit, shower, fire extinguisher, fire blanket, sand etc.)

10. Report to your teacher immediately any significant incident in the laboratory, e.g. injuries, unexpected fires, spills of hazardous chemicals or micro-organisms, or breakages. You are expected to wipe up minor spills yourself, but you must report anything more serious.

11. If you spill a chemical on your skin, eyes or clothing, rinse the affected area immediately with plenty of running water for 15 minutes.

12. You must follow the safety rules for carrying out experiments.

\section{B. Safety rules for carrying out experiments}

\section{Before you start an experiment, you should:}

1. Be familiar with:

- the detailed instructions for the experiment

- the properties and hazards of the chemicals used, how to handle them safely and their risks to health

- the safety precautions for the experiment and the reasons why you need to follow these while performing the experiment;

2. Remember to:

- wear personal protective equipment (eye protection, gloves, lab coats), as instructed by your teacher.

- use the equipment, materials and chemicals as provided by your teacher, and prepare your workplace in accordance with the instructions.

- tie back long hair;

- do not allow ties, scarves, jackets and baggy clothing to hang down loosely.

3. Check that:

- your equipment is not broken or defective;

- your laboratory glassware is clean and free from defects; 
- your chemicals are clearly labelled, including any hazard warnings.

Report any problems to your teacher or the lab technician without delay. Do not use damaged equipment or dirty glassware.

\section{During the experiment:}

4. You should only start the experiment when instructed to do so by your teacher.

5. During the experiment, remain focused on it. Do not distract others with idle chit-chat. If you do need to talk to the teacher or another student about the experiment, do so in a quiet voice.

6. Do not modify the experiment or alter the amount of chemicals used without the prior permission of your teacher.

7. You must strictly follow the rules for basic laboratory operations (e.g. transferring liquids, pipetting, heating - see Appendix 2) and the instructions for using the equipment.

8. Do not leave chemicals in unlabelled containers.

9. Replace the lid or top of all containers immediately after use.

10. If you have any questions concerning the experiment, ASK YOUR TEACHER FOR ADVICE.

\section{After the experiment:}

11. Dispose of all chemical waste according to the instructions. Take particular care with corrosive, toxic, fuming, offensive or reactive chemicals. Never discard solids (including paper, glass, metal and chemicals) into the sink.

12. Rinse laboratory glassware and return it with other equipment and chemicals to its proper place. Wipe down the bench. If you are unable to clean your apparatus, tell your teacher.

13. Make sure that the gas, water, and electricity supplies are turned off.

14. Wash your hands after completing your practical work.

\section{Good laboratory practice for basic operations}

\section{A. Bunsen burner and other naked flames}

1. Before lightning a burner, ensure that there are no flammable substances nearby. Check that the Bunsen burner tubing is tightly connected to the gas supply.

2. Do not lean over any flame! Keep your hands, hair and clothing (especially loose clothing) as well as other flammable materials, away from burners, even if they appear to be turned off?

3. Bunsen burners not in use should have their air-holes closed. If they are not going to be used for a long time, also turn off the gas supply at the mains tap.

4. When lighting a Bunsen burner with a flame, make sure the air-hole is closed. Other types of lighter may require the air-hole to be partly open. Light the gas immediately

\section{B. Heating} after turning on the gas.

1. Never heat any vessel containing flammable liquids directly with a naked flame. Use electric mantles, hot plates or water baths instead.

2. Use appropriate vessels for heating, e.g. round-bottomed flasks, test tubes, beakers, crucibles, evaporating basins. Do not heat thick-walled vessels or pour hot liquids into them.

3. Never heat a closed piece of apparatus.

4. Large volumes of volatile liquids should be heated in a flask fitted with a reflux condenser. Boiling stones (anti-bump granules) must be added before heating commences. Never add boiling stones to a hot liquid. 
5. When heating substances in test tubes, remember:

- not to heat a test tube which is more than $1 / 5$ full

- to point the mouth of the test tube away from yourself and other people

- to lift the test tube in and out of the flame, with frequent shaking, to ensure good mixing and even heating of the whole contents.

- For solids, hold the test tube almost horizontally with a slight upwards tilt.

\section{Transferring liquid substances}

1. Never pipette by mouth! Always use a safety filler. When fitting the filler, hold the pipette one or two centimetres from the end where it is to be fitted.

2. Use a funnel when pouring a liquid from a large container into a narrow-necked container. Ensure there is a gap so that no air lock forms.

3. To avoid drops of liquid flowing down the outside wall of the bottle, use a glass rod when pouring.

\section{Handling dangerous substances}

1. Wear personal protective equipment (eye protection, gloves, laboratory coat), appropriate to the hazards of the substances you are using or making.

2. Use a fume cupboard when the quantities or nature of the hazard requires this; this will often be the case for toxic or corrosive gases and volatile liquids.

3. Dilution of concentrated solutions of acids or bases, and the preparation of such solutions from pure substances is a highly exothermic process. Always stir the solution and cool it in an external water bath as you mix the two.

4. Always add acid to water - in small amounts, with constant stirring - especially when diluting concentrated acids of high density (sulphuric acid).

\section{E. Smelling chemicals}

Never smell chemicals or reaction vessels unless instructed to do so as part of an experiment. Do NOT bring the container directly to your nose. Instead, breathe in to fill the lungs with air. Then point the container away from your face at a distance of about $20 \mathrm{~cm}$, and waft the fumes towards your nose by waving your hand over the container and sniffing gently. Do NOT take deep breaths.

\section{Guidelines for teachers involved in practical science}

Before conducting practical work, make sure that you know what the hazards are, consult any relevant guides and identify any necessary precautions. Your failure to do so may result in accidents.

\section{Remember:}

1. to inspect the gas, electricity, water and ventilation supply regularly as well as the laboratory glassware and equipment;

2. to check if the laboratory or classroom has:

- fire-fighting equipment (fire extinguishers, fire blanket) easily visible and available for immediate use

- $\quad$ personal protective equipment (eye protection, laboratory coats, face shields, gloves etc) available when required

- a chemical spill kit available when required

- $\quad$ eye washing facilities available for immediate use

- means of cutting off gas and electricity supplies in an emergency;

3. to familiarise the students with the chemistry laboratory or classroom rules and the reasons for them. Give them copies and/or post them on the wall;

4. $\quad$ to instruct students in what to do in the event of an accident; 
5. to store chemicals under safe conditions appropriate to their properties. Those which are in any way hazardous must be kept under lock and key in a preparation or store room to which students do not have access;

6. to label clearly all containers of chemicals with the name and hazards of their contents. Do not re-use old containers which formerly contained domestic products;

7. never to leave students unsupervised;

8. that before conducting an experiment:

- you must study the detailed procedure for the experiment, the hazards of the starting materials and products, any risk assessment from your employer, and take all the relevant safety precautions;

- always pre-test the experiment on the same scale and the same conditions under which you intend to do it in class;

- acquaint the students with the detailed procedure for the experiment, the hazards of the starting materials and products and all the relevant safety precautions.

- provide any necessary personal protective equipment

- find out about students' individual medical problems or disabilities (e.g. allergies, asthma, epilepsy and special educational needs)

- inform the students about disposing of chemical waste;

9. that during class demonstrations students must remain at a safe distance. If the demonstration is hazardous, both demonstrator and students should be additionally protected by transparent safety screens;

10. to observe all the principles of good laboratory practice, and expect the same from your students;

11. to ensure that there is adequate Ventilation

Remember that your code of conduct, clothes and behaviour should be a model to your students!

Product of Leonardo da Vinci project CHLASTS Chemical Laboratory Safety Training System 2002-2005, coordinated by Faculty of Chemistry, Jagiellonian University, Kraków, Poland 